\title{
Video Event Recognition for Aircraft Activity Monitoring
}

\author{
David Thirde, Mark Borg, \\ James Ferryman \\ Computational Vision Group \\ The University of Reading \\ Whiteknights, Reading, RG6 6AY, UK \\ \{D.J.Thirde, M.Borg, \\ J.M.Ferryman\}@rdg.ac.uk
}

\author{
Florent Fusier, Valéry Valentin, \\ François Brémond, Monique Thonnat \\ ORION Team \\ INRIA Sophia-Antipolis, 2004 Route des \\ Lucioles BP 93 \\ 06902 SOPHIA-ANTIPOLIS, FRANCE \\ \{Valery.Valentin, Florent.Fusier, \\ Francois.Bremond, \\ Monique. Thonnat\} d sophia.inria.fr
}

\begin{abstract}
This paper presents work in progress on automatic scene interpretation of airport aprons based on a multi-camera video surveillance system. The Scene Tracking and Scene Understanding modules are described and preliminary results and evaluation are presented.
\end{abstract}

\section{INTRODUCTION}

Scene Tracking consists of motion detection and tracking of objects in dynamic scenes over time. Prior work in scene tracking on the apron used a top-down model based approach [1]; such methods are generally computationally expensive and not generic enough for real-time applications. Scene Understanding consists in analysing tracking results both spatially and temporally to automatically recognise the activities occurring in an observed scene. For aircraft servicing analysis, these activities occur around parked aircraft in apron areas. Recent work in scene understanding by Xiang et al [2] applied a hierarchical dynamic Bayesian network to model and hence recognise scene events; however, such models are incapable of recognising simultaneous complex scene activities in real-time over extended time periods.

This paper presents work in progress on the EU project AVITRACK. The architecture employed is a decentralised 8 camera system with overlapping fields of view. Section II details the bottom-up per-camera Scene Tracking module and the data fusion of the tracking results from the camera agents. Section III describes the Scene Understanding, including both the representation and the recognition of video events and the application to apron monitoring.

\section{SCENE TRACKING}

The Scene Tracking module is responsible for the detection and tracking of moving objects from individual cameras; object locations are subsequently transformed into 3D world co-ordinates. The data fusion algorithm then determines single world measurements from the multiple camera observations.

\section{A. Motion Detection}

The output of a motion detector is generally used to find foreground regions (e.g. connected components), which are then used by tracking algorithms to track objects of interest across multiple frames. The airport apron, being an outdoor environment, provides several challenges to motion detection. It must handle a wide range of environmental conditions, including weather and illumination changes, which can be long-term changes (diurnal cycle) or short-term (cloud movements, reflections, etc). 16 motion detection algorithms were implemented and evaluated for the project. Of these, 5 algorithms were found to perform adequately on a range of test sequences: linear prediction-based method [3], mixture of Gaussians [4], colour and edge fusion method [5], kernel density estimation [6], and colour mean and variance. A representative output of each algorithm is shown in Figure 1.

All these methods had acceptable susceptibility to noise, although detection noise was encountered on thin object components (e.g. the aircraft wing edge) due to aliasing and JPEG artifacts. The algorithms were reasonably robust to illumination changes; they were modified with a shadow/highlight detection component based on the work of Horprasert et al [7]. The colour and edge fusion technique was the most sensitive for detection in low contrast regions; linear prediction also had a good detection sensitivity, often finding moving object regions undetected by the other techniques. The most computationally efficient algorithms were the colour mean and variance and the Gaussian mixture model. By taking into account both processing efficiency and sensitivity, the colour mean and variance was selected for the AVITRACK system.

Figure 1 demonstrates the common failing of the detection algorithms tested; with false negatives due to the similar appearance (at pixel level) of the aircraft body and background, and false positives detected in shadowed background regions. The false negatives generally have negligible effect on the estimated bounding box dimensions, since edges of moving object regions are mostly detected. False positives (caused by strong shadow) present a much greater challenge since existing shadow/highlight detection methods generally rely on colour information. AVITRACK datasets contain predominantly achromatic regions (moving and stationary) causing such methods to fail. 


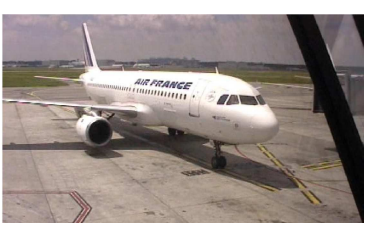

(a)

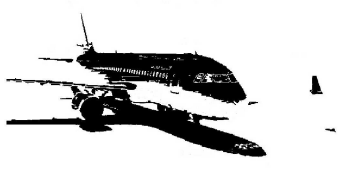

(c)

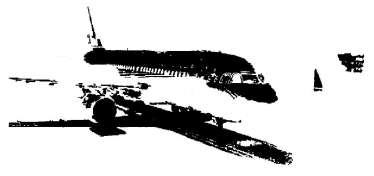

(e)

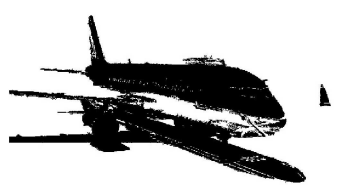

(b)

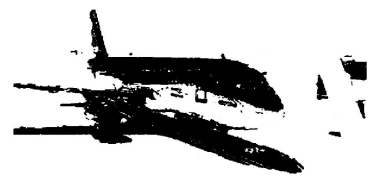

(d)

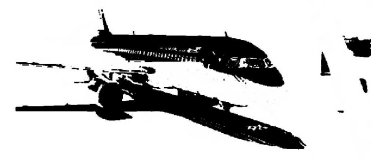

$(f)$
Fig. 1. (a) Observed video frame and (b-f) the motion detection results for frame 1500 taken from the sequence 'Airport03062004', Camera 3. The motion detection algorithms used are: (b) linear prediction, (c) Gaussian mixture model, (d) colour \& edge fusion, (e) kernel density estimation, and (f) colour mean and variance.

\section{B. Object Tracking}

Real-time object tracking can be described as a correspondence problem, and involves finding which object in an image frame relates to which object in the next video frame. Normally, the time interval between two successive frames is small, meaning that inter-frame changes should be limited, thus allowing the use of temporal constraints and/or object features to simplify the correspondence problem. Tracking algorithms have to deal with motion detection errors and complex object interactions; e.g. object merging, dynamic occlusion, fragmentation, non-rigid motion, etc. Three approaches to bottom-up object tracking have been applied in the AVITRACK project: based on the tracking of local features, colour information, and difference image clusters.

The Kanade-Lucas-Tomasi (KLT) feature tracker (described in [8]) combines a local feature selection criterion with local feature matching in adjacent frames. The colour based tracker uses an object's histogram as the global colour model for tracking, and is an adaptation of the CamShift algorithm [9]. The difference image clusters algorithm is based on the work of Pece [10], and uses a probabilistic generative model for detecting and tracking objects.

The KLT algorithm considers local features to be independent entities and tracks each of them individually. Therefore, it is incorporated into a higher-level tracking process that groups features into objects, maintain associations between them, and uses the individual feature tracking results to track objects, taking into account complex object interactions. For each object $O$, a set of sparse features $S$ is mantained, with the number of features determined dynamically from the object size and a configurable feature density parameter $\boldsymbol{\rho}$.
Given a list of tracked objects $\left\{O_{i}^{t-1}\right\}$ at time $t-1$, and a list of observations $\left\{M_{j}^{t}\right\}$ at time $t$ obtained from the motion detector, i.e. connected components of foreground pixels, the tracking process is summarised as:

1) Generate object predictions $\left\{P_{i}^{t}\right\}$ for time $t$ from the list of known objects $\left\{O_{i}^{t-1}\right\}$ at $t-1$.

2) Run the KLT algorithm to track each local feature from the set of features $S_{P_{i}^{t}}$ of prediction $\left\{P_{i}^{t}\right\}$.

3) Given a list of observations $\left\{M_{j}^{t}\right\}$ detected by the motion detector, match predictions $\left\{P_{i}^{t}\right\}$ to observations.

4) Any remaining unmatched predictions in $\left\{P_{i}^{t}\right\}$ are marked as missing observations. Any remaining unmatched observations in $\left\{M_{j}^{t}\right\}$ are considered to be potential new objects.

5) Update the state of those predictions in $\left\{P_{i}^{t}\right\}$ that were matched to observations and replace lost features. The final result is a list of tracked objects $\left\{O_{i}^{t}\right\}$ at time $t$. Let $t=t+1$ and repeat step 1 .

A match function is defined (used for the matching in step 3 above) which returns the number of features $W$ of prediction $P_{i}^{t}$ that reside in the foreground region of observation $M_{j}^{t}$.

$$
f\left(P_{i}^{t}, M_{j}^{t}\right)=\left|\left\{W: W \in S_{P_{i}^{t}}, W \in M_{j}^{t}\right\}\right|
$$

For a non-interacting object, (1) returns a non-zero value for only one prediction and observation. To handle complex object interactactions, a rule-based approach is adopted. The first rule handles the the object splitting case, i.e. several observations at time $t$ match with a single prediction $P_{i}^{t}$. The prediction $P_{i}^{t}$ is then split into new objects, one for each of the matched observations, and the features are assigned to the corresponding new object in which they reside in. In this way, features are maintained through an object splitting event. The second rule handles merging objects, where multiple predictions match with a single observation. As the state of the predictions cannot be obtained by a straightforward update from the combined observation's state, the known local states of the tracked features are used instead.

The local feature tracking method gives the best results, and representative results are shown in Figure 2. It can suffer from loss of object identity if the local features are lost during merging or occlusion and cannot be replenished fast enough. It also requires objects to be textured in order for good features to be selected. With the features being sparse and with a feature density $\rho$ below 0.5 , real-time performance is achieved (12.5 fps). The results of the difference image clusters method look promising, but it suffers from tracking failures when clusters merge together and requires a higher-level process to preserve identities. The colour tracking method performs quite badly because of the achromatic nature of objects in the AVITRACK datasets.

\section{Data Fusion}

The data fusion module combines the tracking data obtained from each of the individual cameras to maximise the useful information content of the scene being observed and hence achieves enhanced occlusion reasoning, a larger visible 
area and improved 3D localisation compared to singlecamera systems. Data fusion also helps to minimise the volume of data generated by the many cameras and reduces the bandwidth needed to send information to later modules. Spatial registration of the cameras is performed using per camera coplanar calibration and the camera streams are synchronised temporally across the network by the central server to prevent temporal drift between the image frames acquired by each camera, which will affect the fusion accuracy.

The current method for data fusion is based on a nearest neighbour Kalman filter approach [11]. The measurement noise covariance $(\mathbf{R})$ is estimated by propagating a (predefined) image plane covariance to the world co-ordinate system at a given value of height for the world location. This estimation of measurement uncertainty allows formal methods to be used to determine the association of observations originating from the same measurement, as well as providing mechanisms for fusing the observations into a single, estimated, measurement. For the measurement covariance $\boldsymbol{\Lambda}$ at location $(x, y)$ in the image plane of camera $c$, the measurement uncertainty $\mathbf{R}\left(x_{w}, y_{w}, z_{w}\right)$ at a given height $z_{w}=0$ (i.e. the ground plane) in the world coordinate system is given by [12] i.e. $\mathbf{R}\left(x_{w}, y_{w}, z_{w}\right)=$ $\mathbf{J}\left(x_{c}, y_{c}\right) \mathbf{\Lambda} \mathbf{J}\left(x_{c}, y_{c}\right)^{T}$

where $\mathbf{J}$ is the Jacobian matrix found by taking the derivatives of the two mapping functions between the image and world co-ordinate systems. The measurement uncertainty field is demonstrated in Figure 2 for camera 6. Note that the uncertainty becomes elongated perpendicular to the sensor in the far-field. For each detected object in the image plane the measurement location $\left(x_{c}, y_{c}\right)$ and associated uncertainty $\boldsymbol{\Lambda}$ is also dependent on the dimensions of the object and a bias can be introduced for larger objects (i.e. vehicles) to increase the uncertainty in the ground plane location of the object; this is currently achieved using a heuristic strategy that incorporates the angle of the camera to the ground plane and the vehicle size. In the data association stage, the per-camera world co-ordinate observations (and associated uncertainty) are matched to existing tracks using the nearest neighbour search strategy.

A validation gate is applied to limit the potential matches in the association step. The gate is determined by a threshold $\tau$ on the normalised innovation squared distance between the predicted track states and the observed measurements:

$$
d^{2}=\left(\mathbf{H} \widehat{\mathbf{X}}_{k}^{-}-\mathbf{Z}_{k}\right)^{T} \mathbf{S}_{k}^{-1}\left(\mathbf{H} \widehat{\mathbf{X}}_{k}^{-}-\mathbf{Z}_{k}\right)
$$

where $\mathbf{S}_{k}=\mathbf{H} \widehat{\mathbf{P}}_{k}^{-} \mathbf{H}^{T}+\mathbf{R}_{k}$ is the innovation covariance and $\mathbf{Z}_{k}$ is the observed measurement at time $k$. An appropriate threshold can be determined from standard tables of the chi-square distribution with the degrees of freedom determined by the dimensionality of the measurement vectors. For matched observations, the location and uncertainties are combined to find the optimal fused estimate of the location and uncertainty of the object. This is achieved using two methods - covariance accumulation and covariance intersection. Covariance accumulation estimates the fused

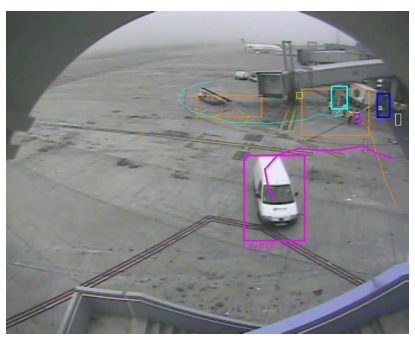

(a)

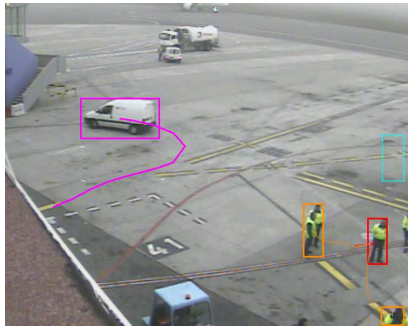

$(c)$

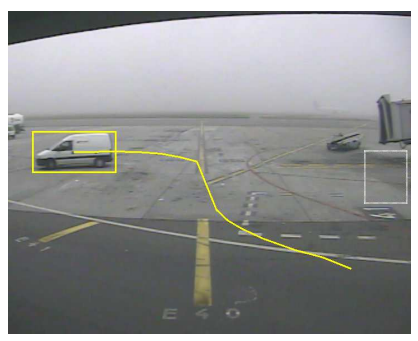

(b)

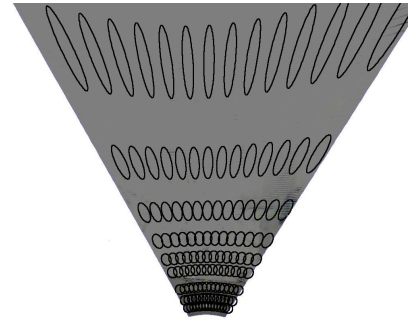

(d)

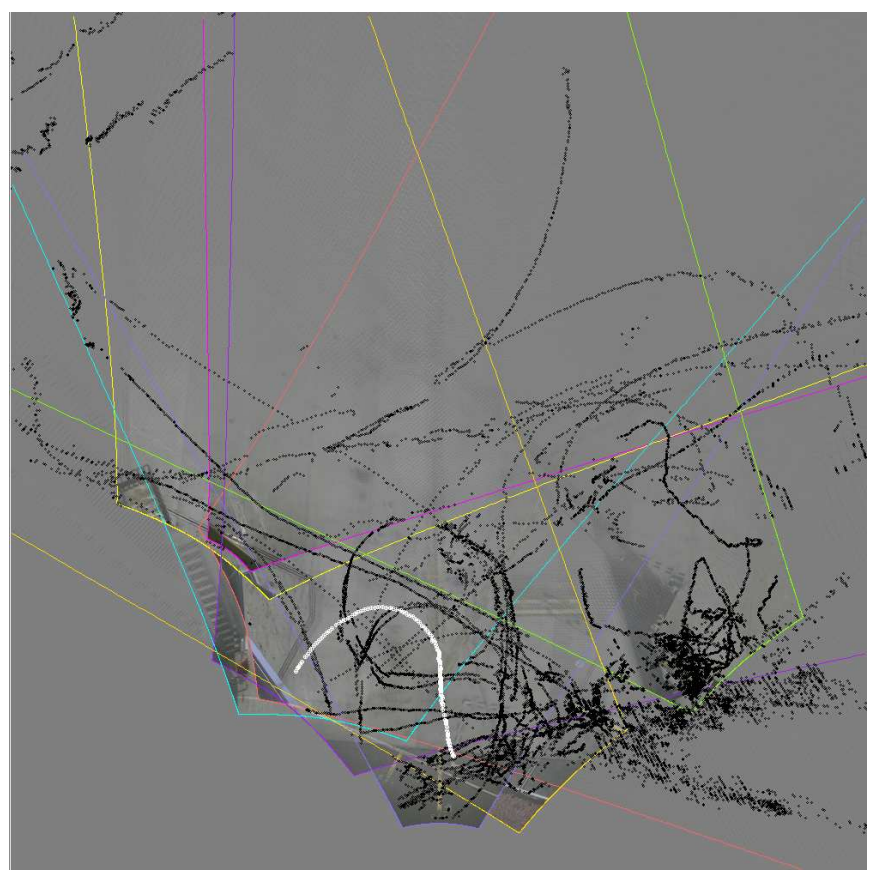

$(e)$

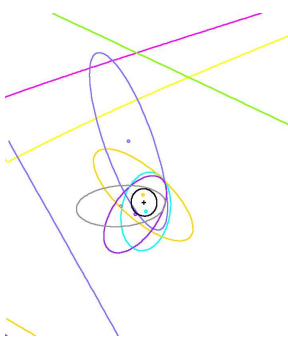

$(f)$

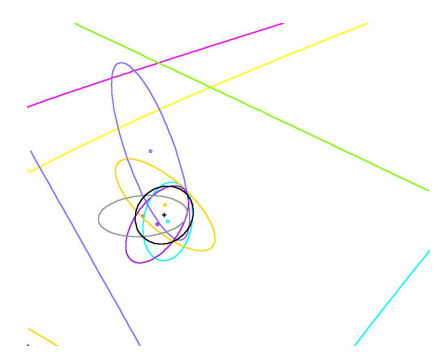

$(g)$
Fig. 2. (a-c) The tracking results for 3 cameras for frame 9126 of sequence 21 are shown; image (d) shows the sensory uncertainty fi eld measured for camera 6, and plotted on to the ground-plane; fi gure (e) shows the data fusion results on the ground-plane for the sequence (9600 frames), with the services vehicle track highlighted in white; fi gure (f) shows the fused observation (in black) for the services vehicle using the covariance accumulation method, while image (g) shows the result for the covariance intersection method. 
uncertainty $\mathbf{R}_{\text {fused }}$ for $N$ matched observations as $\mathbf{R}_{\text {fused }}=$ $\left(\mathbf{R}_{1}^{-1}+\ldots+\mathbf{R}_{N}^{-1}\right)^{-1}$. The covariance intersection method is conceptually similar except that the observation uncertainty covariances are weighted in the summation:

$$
\begin{gathered}
\mathbf{R}_{\text {fused }}=\left(w_{1} \mathbf{R}_{1}^{-1}+\ldots+w_{N} \mathbf{R}_{N}^{-1}\right)^{-1} \\
\text { where weight } t_{i}=\frac{w_{i}^{\prime}}{\sum_{i=1}^{N} w_{i}^{\prime}} \text { and } w_{i}^{\prime}=\frac{1}{\operatorname{Tr}\left(\mathbf{R}_{i}^{c}\right)}
\end{gathered}
$$

and $\mathbf{R}_{i}^{c}$ is the measurement uncertainty of the $i$ 'th associated observation (made by camera $c$ ); Covariance intersection therefore weights in favour of the sensors that have more certain measurements. The resulting fused observations are demonstrated in Figure 2 for the "Services Vehicle" object; the covariance accumulation method gives a more localised estimate of the fused measurement than the covariance intersection approach. Any remaining unassociated measurements are clustered into new tracks, using a validation gate between observations to constrain the association and fusion steps.

\section{SCENE UNDERSTANDING}

The aim of Scene Understanding is to perform a high level interpretation of the scene observed through video sequences by detecting video events occurring in the scene. To detect these events, we use spatio-temporal reasoning based cognitive vision techniques, a priori knowledge of the observed environment and a set of predefined event models. The Scene Understanding task is based on a Video Event Recognition module that takes the tracked mobile objects from the previously described modules as input, and generates recognised events as output. The Scene Understanding task is processed in real time, from 12.5 to 15 frames per second.

\section{A. A Priori Knowledge}

The a priori knowledge of the environment corresponds to a 3D empty scene model of the observed environment and a set of video events models. The empty scene model contains static information about the contextual objects (e.g. equipments, zones of interest, airport walls, jet-bridge) characterised either by their 3D geometry (approximative shape) and semantics (e.g. how they interact with operating people). The video event models are predefined by experts of the domain (e.g. managers of handling companies) using a video event description language described in [13]. The video event models are manually defined and some works are in progress to upgrade this process to learn the video events from video sequences directly.

\section{B. Video Event Representation}

The goal of video event representation is to formalise the knowledge for the system to be able to detect video events occurring in the observed scene. The description of this knowledge has to be declarative and intuitive (in natural terms), so that the experts in the aircraft activity monitoring can easily define and modify it. A video event model $\mathrm{E}$ is composed of five components:

- a set of Physical Object variables corresponding to the physical objects involved in E (any contextual objects including static objects (equipment, zones of interest) and mobile objects (people, vehicles, aircraft... categorised and tracked by the Scene Tracking module))

- a set of temporal variables corresponding to the components (sub-events) of E

- a set of forbidden variables corresponding to the components that are not allowed to occur during the detection of $\mathrm{E}$

- a set of constraints (symbolic, logical, spatial and temporal constraints including Allen's interval algebra operators[14]) involving these variables

- a set of decisions corresponding to the tasks predefined by experts that are needed to be executed when $E$ has been detected (e.g. to launch an alarm or to display a message on a window)

There are four types of video events: primitive states, composite states, primitive events and composite events. A state describes a situation characterising one or several physical objects defined at time $t$ or a stable situation defined over a time interval. A primitive state (e.g. a person is located inside a zone) corresponds to a vision property directly computed by the vision module. A composite state as shown in Figure 3 corresponds to a combination of primitive states.

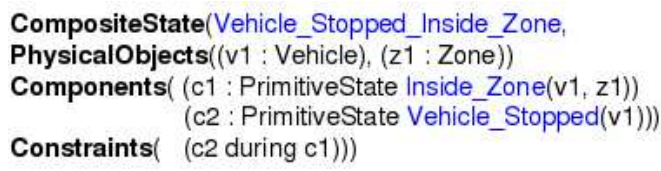

Fig. 3. The model of the composite state "Vehicle_Stopped_Inside_Zone" is composed of two components and one constraint.

An event is an activity containing at least a change of state values between two consecutive times (e.g. a vehicle enters a zone of interest: it is outside the zone and then inside the zone). A primitive event (as shown in Figure 4) is a change of primitive state values and a composite event is a combination of states and/or events.

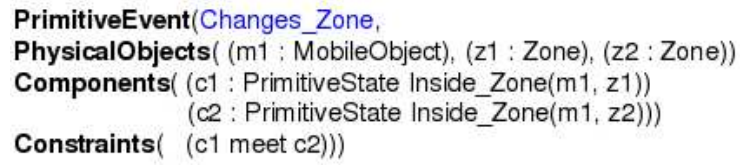

Fig. 4. The model of the primitive event "Changes_Zone" is composed of two components and one constraint.

\section{Video Event Recognition}

The video event recognition algorithm recognises which events are occurring in a stream of mobile objects tracked by the Scene Tracking module. The algorithm to recognise a primitive state consists in a loop of two operations: (1) selection of a set of physical objects then (2) verification of the corresponding atemporal constraints until all combinations of physical objects have been tested. Once a set of physical objects satisfies all atemporal constraints, the primitive state is said to be recognised. In order to facilitate primitive event 
recognition, event templates are generated for each primitive event the last component of which corresponds to this recognised primitive state. The event template contains the list of physical objects involved in the primitive state. These physical objects partially instantiate the event template.

To recognise a primitive event given the event template partially instantiated, the recognition algorithm consists in selecting (if needed) a set of physical objects matching the remaining physical object variables of the event model then looking backward in the past if a previously recognised primitive state matches the first component of the event model. If these two recognised components verify the event model constraints, the primitive event is said to be recognised. In order to facilitate composite event recognition, after each primitive event recognition, event templates are generated for all composite events the last component of which corresponds to this recognised primitive event.

The recognition of composed states and events usually implies a search in a large space composed of all the possible combinations of components and physical objects. To avoid this combinatorial explosion, all composed states and events are decomposed into states and events composed at the most of two components through a stage of compilation in a preprocessing phase. Then the recognition of composed states and events is performed similarly to the recognition of primitive events. The video event recognition algorithm is detailed in [15].

\section{SCENE UNDERSTANDING FOR APRON MONITORING}

\section{A. Dynamic contextual information}

In the Apron Monitoring application, the contextual information of the empty scene model containing the contextual objects (e.g. equipments, zones of interest like access or stopping zones) is necessary. This is static and fixed contextual information. Dynamic contextual information about vehicles (e.g. Ground Power Unit (GPU), Loader, Tanker) and aircraft is needed and is therefore defined in the local coordinate system of the vehicles and aircraft. To compute this dynamic contextual information in the global coordinate system, the context manager module computes a transformation matrix using the orientation and the position of the tracked mobile objects. Then, for parked vehicles and aircraft, the dynamic zones in the global coordinate system are added into the static context (empty scene model) to recognise people and vehicle interactions. This allows, for example, the recognition that a person exits a vehicle (e.g. the person appears in the dynamic zone corresponding to the vehicle door which has been added to the empty scene model when the vehicle has been parked as shown on Figure 5.)

\section{B. Predefined Video Events}

In the current work a generic set of 5 primitive states, 4 composite states and 3 primitive events needed for the recognition of handling operations has been defined. In addition to these generic video events, emphasis has been placed on handling operations which only involves a vehicle and/or a

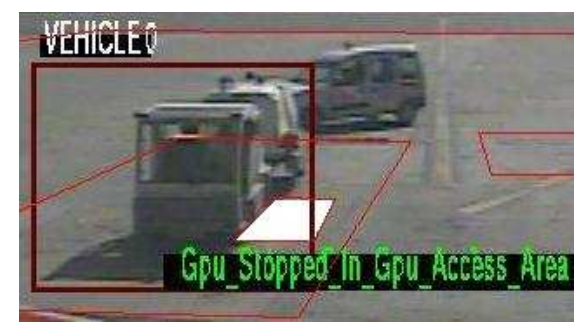

Fig. 5. Dynamic zone representing the vehicle door added in the empty scene model after the stop of the vehicle.

person. The main test was performed for the "Aircraft Arrival Preparation" event. This operation involves a vehicle (GPU), its driver (Handler) and four zones of interest. The system recognises that the GPU arrives and stops in the access area and then the driver gets out from the GPU and deposits the chocks and the stud at the location where the plane will stop. This operation (as shown in Figure 6) is recognised when the 5 video events involving the GPU have been recognised and the constraints verified.

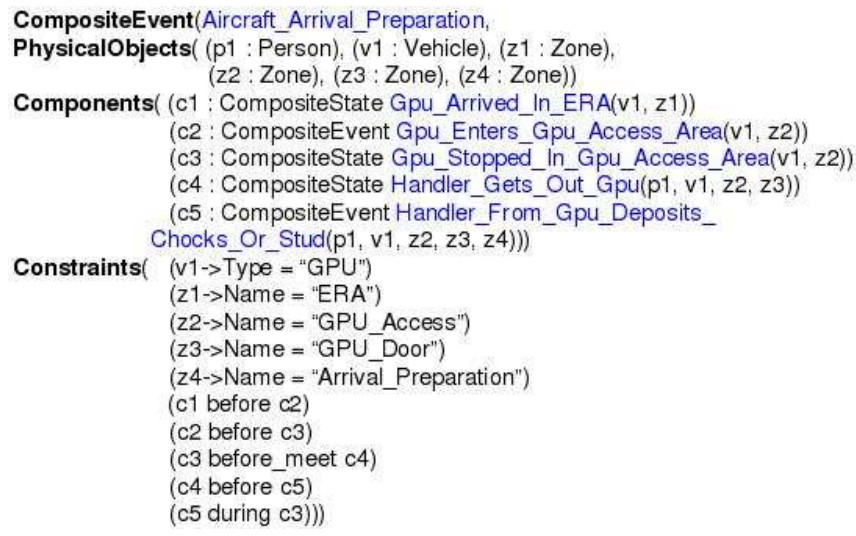

Fig. 6. The model of the composite event "Aircraft_Arrival_Preparation" contains 6 physical objects, 5 components and 10 constraints.

We have also modelled the video event corresponding to the "Refueling Operation". The system has to recognise that the Tanker arrives and stops in the "Refueling Area" and then the driver exits from the vehicle and refuels the aircraft. The video event recognition module has been tested on a first stage of the "Refueling Operation" corresponding to the part when the Tanker is getting ready to refuel the aircraft. The model of the composite event "Tanker_Arrival" contains 3 physical objects, 3 components and 5 constraints.

\section{Results}

In this section the evaluation of the Scene Understanding module is considered. The first evaluation was undertaken on the sequences on which the Scene Tracking module gives good results. The video events involving a GPU have been tested on a dataset of 4 scenes corresponding to 8 video sequences (containing from 1899 to 3774 frames and including one night sequence) showing the "Aircraft Arrival Preparation" and 2 scenes showing the "Tanker Arrival" (see 
TABLE I

TP = "EVENT EXISTS IN THE REAL WORLD AND IS WELL RECOGNISED", FN = "EVENT EXISTS IN THE REAL WORLD BUT IS NOT RECOGNISED", FP = "EVENT DOES NOT EXIST IN THE REAL WORLD BUT IS RECOGNISED"

\begin{tabular}{|c|c|c|c|c|}
\hline Event & Sequences & TP & $\mathbf{F P}$ & $\overline{\text { FN }}$ \\
\hline \multicolumn{5}{|l|}{ GPU } \\
\hline Event 1 & 4 scenes $* 2$ cam. & 8 & 0 & 0 \\
\hline Event 2 & 4 scenes $* 2$ cam. & 8 & 0 & 0 \\
\hline Event 3 & 4 scenes. $* 2$ cam. & 8 & 0 & 0 \\
\hline Event 4 & 4 scenes. $* 2$ cam. & 8 & 0 & 0 \\
\hline Event 5 & 2 scenes $* 1$ cam. & 2 & 3 & 0 \\
\hline Event 6 & 2 scenes $* 1$ cam. & 2 & 0 & 0 \\
\hline Event 7 & 2 scenes $* 1$ cam. & 2 & 0 & 0 \\
\hline Event 8 & 2 scenes $* 1$ cam & 2 & 0 & $\overline{0}$ \\
\hline \multicolumn{5}{|l|}{ Tanker } \\
\hline Event 9 & 2 scenes $* 1$ cam. & 2 & 0 & 0 \\
\hline Event 10 & 2 scenes $* 1$ cam. & 2 & 0 & 0 \\
\hline Event 11 & 2 scenes $* 1$ cam. & 2 & 0 & 0 \\
\hline Event 12 & 2 scenes $* 1$ cam. & 2 & 0 & $\overline{0}$ \\
\hline Event 13 & 2 scenes $* 1$ cam. & 2 & 0 & 0 \\
\hline
\end{tabular}

Table I). The Scene Understanding module has been tested on the two best points of view from where the GPU can be observed and on the only point of view from where the Tanker can be observed.

The current evaluation is mainly qualitative and performed manually with no ground truth. The goal is only to provide an idea of the performance of the Scene Understanding and to anticipate potential problems in event detection for apron monitoring. The result of the performance evaluation shows that all video events are correctly recognised (45 TPs) with very few false alarms ( $3 \mathrm{FPs}$ ) and no miss detection (0 FNs). The results are very encouraging but it must be considered that situations in which the vision module misdetects or overdetects mobile objects are not yet addressed. The events $5,6,7$ and 8 are only detected on one of the two tested cameras because this part of the scene is only observed by one camera. The FPs of event 5 are due to too vague a model of event 5 . This event should be detected when the handler (driver of the GPU vehicle) exits from the vehicle in a predefined zone near the door of the vehicle (called "Gpu_Door"). However, at present, this event is detected when a person is located inside this zone and it is analysed by the system as the person is exiting from the GPU vehicle.

\section{CONCLUSIONS AND FUTURE WORK}

\section{A. Conclusions}

The preliminary results are encouraging for both the Scene Tracking and Understanding modules. The performance of multi-view object tracking provides adequate results; however, tracking is sensitive to significant dynamic and static object occlusion within the scene. The video event recognition results prove that Scene Understanding can be applied to apron monitoring. The main difficulty in using the video event recognition module for apron monitoring is to model the handling operations using expert knowledge (25 video events already defined) and to try to recognise them all in parallel and in real time.

\section{B. Future Work}

Future work in Scene Tracking will address effective shadow supression, explicit occlusion analysis, and improved data fusion. Future work in Scene Understanding will consider also the recognition of more complex operations (e.g. "baggage loading/unloading") involving more people and vehicles. Further planned work includes modelling uncertainty to enable recognition of events even when the Scene Tracking module loses physical objects or gives unreliable output.

\section{ACKNOWLEDGMENTS}

This work was supported by the European Union, grant AVITRACK (AST3-CT-3002-502818) ${ }^{1}$. The AVITRACK website is located at www.avitrack.net.

\section{REFERENCES}

[1] Sullivan, G. D. "Visual Interpretation of known objects in constrained scenes" Phil. Trans. R. Soc. Lon., B, 337, pp 361370, 1992

[2] T. Xiang and S. Gong, "On the Structure of Dynamic Bayesian Networks for Complex Scene Modelling." In Proc. Joint IEEE International Workshop on Visual Surveillance and Performance Evaluation of Tracking and Surveillance (VS-PETS), pp 17-22, Nice, France, October, 2003.

[3] K. Toyama, J. Krumm, B. Brumitt, and B. Meyers, 'Wallflower: Principles and practice of background maintenance." In Proc. International Conference on Computer Vision, pp 255-261, 1999.

[4] C. Stauffer and W.E.L. Grimson, "Adaptive Background Mixture Models for Real-Time Tracking." In Proc. International Conference on Pattern Recognition, pp 246-252, 1999.

[5] S. Jabri, Z. Duric, H. Wechsler and A. Rosenfeld, 'Detection and Location of People in Video Images Using Adaptive Fusion of Color and Edge Information." In Proc. International Conference on Pattern Recognition, pp 4627-4631, 2000.

[6] A. Elgammal, D. Harwood and L. Davis, "Non-parametric model for background subtraction." In IEEE ICCV'99 FRAME-RATE WORKSHOP, 1999.

[7] T. Horprasert, D. Harwood and L. S. Davis, "A statistical approach for real-time robust background subtraction and shadow detection." In IEEE International Conference on Computer Vision, FRAME-RATE WORKSHOP, 1999.

[8] J. Shi and C. Tomasi, "Good Features to Track." In Proc. of IEEE Conference on Computer Vision and Pattern Recognition, pp 593-600, 1994.

[9] G. Bradski, "Computer Vision Face Tracking For Use in a Perceptual User Interface.” Intel Technology Journal, Q2, 1998.

[10] A. E. C. Pece, 'From Cluster Tracking to People Counting." In IEEE Workshop on Performance Evaluation of Tracking and Surveillance, pp 9-17, 2002.

[11] Y. Bar-Shalom and X. R. Li, Multitarget-Multisensor Tracking: Principles and Techniques, YBS Publishing, 1995.

[12] J. Black and T. J. Ellis, "Multi Camera Image Measurement and Correspondence." Measurement - Journal of the International Measurement Confederation, 35(1) July, pp 61-71, 2002.

[13] F. Br'emond, N. Maillot, M. Thonnat and V. Vu. 'Ontologies for Video Events." Research report number 51895, INRIA Sophia-Antipolis, Nov 2003.

\footnotetext{
${ }^{1}$ However, this paper does not necessarily represent the opinion of the European Community, and the European Community is not responsible for any use which may be made of its contents.
} 
[14] J. F. Allen. 'Maintaining Knowledge about Temporal Intervals." In Communications of the ACM, 26(11) pp 823-843, Nov 1983.

[15] V. Vu, F. Br'emond, M. Thonnat, "Automatic Video Interpretation: A Novel Algorithm for Temporal Event Recognition." IJCAI'03, Acapulco, Mexico, 9-15 Aug 2003. 